\title{
3D Multi-branch Tubular Surface and Centerline Extraction with 4D Iterative Key Points
}

\author{
Hua $\mathrm{Li}^{1}$, Anthony Yezzi ${ }^{2}$, and Laurent Cohen ${ }^{3}$ \\ 1 Department of Radiology, Mayo Clinic College of Medicine, Rochester, MN 55905, USA \\ 2 School of ECE, Georgia Institute of Technology, Atlanta, GA 30332, USA \\ 3 CEREMADE, CNRS UMR 7534, University Paris-Dauphine, 75775 Paris Cedex, France
}

\begin{abstract}
An innovative 3D multi-branch tubular structure and centerline extraction method is proposed in this paper. In contrast to classical minimal path techniques that can only detect a single curve between two pre-defined initial points, this method propagates outward from only one initial seed point to detect 3D multi-branch tubular surfaces and centerlines simultaneously. First, instead of only representing the trajectory of a tubular structure as a 3D curve, the surface of the entire structure is represented as a 4D curve along which every point represents a 3D sphere inside the tubular structure. Then, from any given sphere inside the tubular structure, a novel 4D iterative key point searching scheme is applied, in which the minimal action map and the Euclidean length map are calculated with a 4D freezing fast marching evolution. A set of 4D key points is obtained during the front propagation process. Finally, by sliding back from each key point to the previous one via the minimal action map until all the key points are visited, we are able to fully obtain global minimizing multi-branch tubular surfaces. An additional immediate benefit of this method is a natural notion of a multi-branch tube's "central curve" by taking only the first three spatial coordinates of the detected 4D multi-branch curve. Experimental results on 2D/3D medical vascular images illustrate the benefits of this method.
\end{abstract}

\section{Introduction}

Image segmentation is often the first task for solving problems in the fields of image processing and computer vision. In medical imaging, the extraction of vascular objects such as coronary arteries and retinal blood vessels, has attracted the attention of more and more researchers [1,2]. Numerous segmentation methods have been proposed that depend upon organ structures, imaging modalities, application domains, user-interaction requirements, and so on [3].

Centerline extraction methods have been proposed to extract only the centerline (or skeleton) of a tubular object, thereby requiring further processing to obtain the 3D surface or shape. By assuming a centerline corresponded to a minimal cost path, some methods have been designed based on path finding procedures [4, 5, 6, 7, 8, 9, 10]. Specially, Deschamps and Cohen [11] simplified the problem of generating centerlines into the problem of finding minimal paths [12] by utilizing fast marching schemes [13]. The minimal path approach [12] has several advantages such as finding global minimizers, fast computation, ease of implementation, and powerful incorporation of user input.

G.-Z. Yang et al. (Eds.): MICCAI 2009, Part II, LNCS 5762, pp. 1042-1050, 2009.

(C) Springer-Verlag Berlin Heidelberg 2009 
Unfortunately, despite these numerous advantages, traditional minimal path techniques exhibit some disadvantages both in general and in the particular application of vessel segmentation [14]. First, follow-up vessel boundary extraction is by no means straight forward, even in 2D where the longitudinal cross-sectional boundary of a vessel is completely described by two curves on either side of the detected trajectory. Second, the detected interior trajectory does not always yield remain central to the vessel. Third, in 3D (just as in 2D), traditional purely spatial minimal path techniques can be used only for curve extraction, whereas vessels and other tubular structures, despite sharing some characteristics with curves, are in fact surfaces. Finally, only a single branch can be detected for each pair of initializing seed points. Multiple initialization pairs would be required in order to detect multi-branch structures.

As an improvement, Li and Yezzi [14] proposed a 4D minimal path technique to extract full 3D tubular surfaces and their centerlines simultaneously. They represented the surface of a tubular structure as the envelope of a one-parameter family (curve) of spheres with different centers (three coordinates) and different radii (fourth coordinate). So the $3 D$ surface extraction problem is translated into the problem of finding a $4 D$ curve which encodes this family of $3 \mathrm{D}$ spheres. As such, the tubular surface and its centerline can be detected simultaneously in this one-dimension-higher 4D space. However, in their method, the user input is still a pair of initial points (or spheres), and thus only a single branch can be detected for each pair of initializations. This disadvantage significantly limits its application to most vessels which have complex branching topologies such as the coronary arteries and abdominal aorta.

In this paper, we propose an innovative 3D multi-branch tubular structure and centerline extraction method with all the merits of minimal path techniques while further limiting the required user-interaction to a single initial point. By modeling the surface as a 4D trajectory as in [14], a set of branching 3D tubular surfaces and their centerlines are simultaneously detected with a guarantee that the centerline curves also connect at branch points. By starting with a single 4D point (i.e. a single sphere inside the 3D vessel or other tubular structure) a novel scheme is applied to find a set of 4D iterative key points along an optimal 4D path which is free to branch whenever it is energetically favorable. Then, by sliding back from each key point to the previous one along the minimal action map until all the key points are visited, we obtain the final global minimizing multi-branch 4D trajectory from which we may directly construct the branching tubular 3D surface along with its 3D "central curve", both of which are guaranteed to exhibit the exact same branching topology. Experimental results on 2D/3D medical vascular images illustrate the benefits of this method.

\section{Multi-branch Tubular Structure Extraction}

When only a single initial starting point is provided by the user, detecting an optimal branching trajectory using minimal cost path searching schemes is dependent upon finding one or more appropriate destination points on the desired path. Furthermore, in order to detect multi-branch structures, at least one destination point along each individual branch should be found. We may then slide back from each destination point toward the starting point to obtain the multi-branch tubular structure. Here, we propose a 4D key point searching scheme to carry out these tasks. 


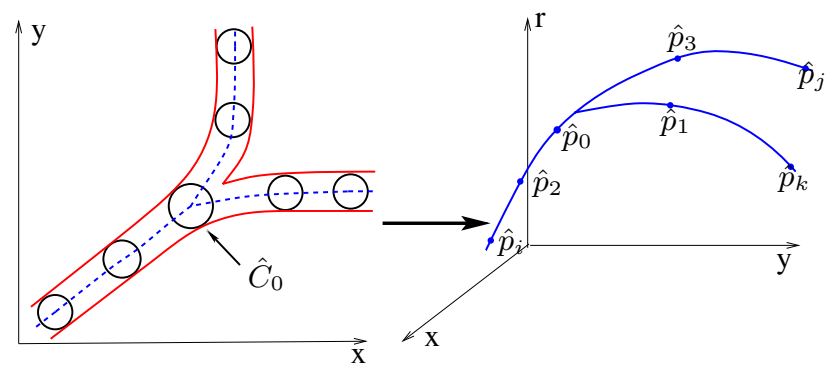

Fig. 1. The entire multi-branch structure extraction is reduced to finding structures between all adjacent key point pairs. The 4D path length $D$ between each key point pair is equal to $d_{\text {step }}$. For easier visualization, the same concept is illustrated here using circles instead of spheres.

\subsection{D Key Point Searching Scheme}

Motivated by the 4D minimal path technique [14], we first represent the entire vessel as a $4 \mathrm{D}$ curve, where each $4 \mathrm{D}$ point represents a $3 \mathrm{D}$ sphere (three coordinates for the center point and one for the radius). The $3 D$ surface extraction problem is translated into the problem of finding a $4 \mathrm{D}$ curve which encodes this family of 3D spheres.

For detecting multi-branch tubular structures, the new 4D iterative key point searching scheme is proposed and illustrated in Fig. 1 The energy minimization model is

$$
E(\hat{C})=\int_{\Omega}\{\omega+P(\hat{C}(c(s), r(s)))\} d s=\int_{\Omega} \widetilde{P}(\hat{C}) d s,
$$

where $s$ represents the arc-length parameter over an interval $\Omega, c(s)$ represents the location of a point in the original image domain $\Omega_{I}$ either in $\mathbb{R}^{2}[c(s)=(x(s), y(s))]$ or in $\mathbb{R}^{3}[c(s)=(x(s), y(s), z(s))], r(s) \in\left[0, r_{\max }\right]$ represents the radius of a circle/sphere centered at $c(s)\left(r_{\max }\right.$ is the largest allowed thickness of the vessel to be captured), $\hat{C}(c(s), r(s)) \in \Omega_{I}$ represents a multi-branch "path" composed by a family of circles/spheres in $\Omega_{I}, E(\hat{C})$ represents the energy which is the integral of $\widetilde{P}$ along $\hat{C}(c(s), r(s)), P$ is the potential that enhances the boundary of the vascular tree, $\omega$ is a real positive constant, and $\widetilde{P}=P+w$. We call $\Omega_{I_{r}}=\Omega_{I} \times\left[0, r_{\text {max }}\right]$.

Alternatively, as shown in Fig. 11, energy $E$ along the entire structure can be treated as the summation of energy $E_{k}$ along a path $\hat{C}_{k}$ between every two adjacent key points $\hat{p}_{k_{0}}$ (as the starting point)and $\hat{p}_{k}$ (as the ending point),

$$
E(\hat{C})=\sum_{k=1}^{N} E_{k}\left(\hat{C}_{k}\right)=\sum_{k=1}^{N}\left(\int_{\Omega} \widetilde{P}_{k}\left(\hat{C}_{k}\right) d s\right) .
$$

where $P_{k}$ may be different for finding different minimal cost path $C_{k}$. It is well known that an appropriate $\widetilde{P}_{k}$ with lower values near the optimal path will cause fronts starting from the initial point $\hat{p}_{k_{0}}$ to propagate faster along the desired minimal path. Here we define an additional item: the distance step $d_{\text {step }}$. We choose the first reached point at which a front starting from the initial point $\hat{p}_{0}$ travels $d_{\text {step }}$, and we labeled it as key point $\hat{p}_{1}$. We then trace back the optimal minimal path between it and $\hat{p}_{0}$. The next key 
point $\hat{p}_{2}$ will be the first reached point at which a front, starting simultaneously from $\hat{p}_{0}$ and $\hat{p}_{1}$, travels the distance $d_{\text {step }}$. This will become the destination point for the minimal path segment $\hat{C}_{2}$. Repeating this process, the new key point $\hat{p}_{k}$ is always the first reached point at which a front, starting simultaneously from points $\hat{p}_{0}$ to $\hat{p}_{(k-1)}$, travels the distance $d_{s t e p}$. In this way, the front may propagate to different branches of the tubular structure, and key points along different branches may be obtained. The process stops when no new key point can be found, which is guaranteed by freezing fast marching schemes and will be discussed later.

To find each minimal path segment $\hat{C}_{k}$ (minimizing the energy $E_{k}$ ) between key point pairs $\hat{p}_{k_{0}}$ and $\hat{p}_{k}$ (the starting point $\hat{p}_{k_{0}}$ may be any one of the points $\hat{p}_{0}$ to $\hat{p}_{(k-1)}$ ), the $4 \mathrm{D}$ minimal path technique [14] is applied. At any $4 \mathrm{D}$ point $\hat{p} \in \Omega_{I_{r}}$, we define the minimal action map $U_{k}(\hat{p})$ as the minimal energy integrated along any possible path between the starting point $\hat{p}_{k_{0}}$ and the point $\hat{p}$,

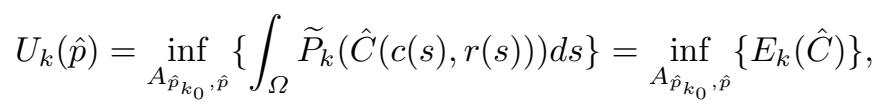

where $A_{\hat{p}_{k_{0}}, \hat{p}}$ is defined as the set of all paths between $\hat{p}_{k_{0}}$ and $\hat{p}$. So, from the ending point $\hat{p}_{k}$, the minimal path between $\hat{p}_{k_{0}}$ and $\hat{p}_{k}$ can be deduced by calculating $U_{k}\left(\hat{p}_{k}\right)$ and then sliding back from $\hat{p}_{k}$ along the action map $U_{k}$ to $\hat{p}_{k_{0}}$ via gradient descent. $U_{k}(\hat{p})$ can be computed by solving the Eikonal equation,

$$
\left\|\nabla U_{k}\right\|=\widetilde{P}_{k}(\hat{p}) \quad \text { with } \quad U_{k}\left(\hat{p}_{k_{0}}\right)=0, \quad \hat{p} \in \Omega_{I_{r}},
$$

using the fast marching algorithm [13]. 3D (or 4D) fast marching schemes are utilized to solve the Eikonal equations and calculate the action maps for 2D (or 3D) spheres respectively. The 3D vessel structure is then obtained as the envelope of the family of spheres traversed along this $4 \mathrm{D}$ curve. Also because all the spheres on the detected minimal path are tangential to the boundary of the tubular structure, the union of their center points describes the central path (medial axis) of the tubular structure.

In order to find appropriate key points and also reduce the computational cost, we would like to limit the front propagation within the long tubular structure. A freezing fast marching method proposed in [15] is utilized to stop the propagation of these fronts when they reach the structure boundary. Obviously, we would like to freeze points at the "tail" of the front, especially when it reaches the actual boundary of tubular shape, and keep the points at the "head" of the front propagating further. To be able to distinguish points at the "head" from those at the "tail", the path lengths $D_{k}(\hat{p})$ from the starting point $\hat{p}_{k_{0}}$ to any other point $\hat{p}$ should be computed. $D_{k}(\hat{p})$ is the Euclidean distance traveled by the front from the starting point $\hat{p}_{k_{0}}$ to any other point $\hat{p}$. When $D_{k}(\hat{p})$ is smaller than the current maximum path length $d_{\max }$ (initial $d_{\max }=0$ ),

$$
D_{k}(\hat{p})<\max \left(\left(d_{\max }-\widetilde{d}\right), 0\right) \text { with } \tilde{d}>0,
$$

point $\hat{p}$ is frozen by setting the speed to 0 , where $\widetilde{d}$ is a pre-defined threshold value. Eq. 5 ensures that no point is frozen till one point on the front has traveled at least the Euclidean distance $\widetilde{d}$, which enables the front to stay inside the long and thin structure. 
The distance threshold $\widetilde{d}$ is a parameter which should be larger than the expected maximum branch segment of the object, otherwise the algorithm will wrongly freeze points prematurely within incomplete branches. An appropriate $\widetilde{d}$ should be chosen based on different image qualities, vessel structures, noise levels, and so on. Instead of calculating $D_{k}$ after extracting the minimal path according to gradient descent, we may solve $D_{k}$ locally using the same neighbors involved for solving $U_{k}$ in Eq. 4

$$
\left\{\begin{array}{l}
\left\|\nabla U_{k}\right\|=\widetilde{P}_{k} \quad \text { with } \quad \begin{array}{rl}
U\left(\hat{p}_{k_{0}}\right)=0 \\
\left\|\nabla D_{k}\right\|=1
\end{array} \quad \text { with } \quad D\left(\hat{p}_{k_{0}}\right)=0
\end{array}\right.
$$

Second, since key point $\hat{p}_{k}, k \in\{1, \ldots, N\}$ is the first reached point at which a front, starting simultaneously from multiple points $\hat{p}_{0}$ to $\hat{p}_{(k-1)}$, travels the distance $d_{\text {step }}$, the optimal minimal cost path $\hat{C}_{k}$ should connect $\hat{p}_{k}$ to the appropriate previous key point $\hat{p}_{k_{0}}, k_{0} \in\{0, \ldots,(k-1)\}$. Here we separate the back tracking process into each new key point searching step to avoid false connections. Each time when we obtain a new key point $\hat{p}_{k}$, we slide back right away from $\hat{p}_{k}$ according to the gradient descent on the minimal action map until reaching any point in the group of points $\hat{p}_{0}$ to $\hat{p}_{(k-1)}$ and obtaining the minimal path $\hat{C}_{k}$. Parameter $d_{\text {step }}$ also defines the accuracy and computational cost of the method. If it is big, it decreases the computational cost of tracking the connectivity of the fronts. However, it misses branches shorter than $d_{\text {step }}$.

Furthermore, if the detected tubular structure only has one branch, the 4D iterative process can be simplified to the following process. First, an initial point $\hat{p}_{0}$ should be chosen at the end of the tubular structure in order to detect the whole structure. Similarly, if the front starting from $\hat{p}_{0}$ will travel at least the distance $N \times d_{\text {step }}$, the first reached points when the front travels the distance $n \times d_{\text {step }}, n \in\{1, \ldots, N\}$ are chosen as the labeled key points $\hat{p}_{n}$. The $4 \mathrm{D}$ minimal path between the initial point $\hat{p}_{0}$ and the last iterative key point $\hat{p}_{N}$ can be easily deduced by sliding back from point $\hat{p}_{N}$ to its previous point $\hat{p}_{(N-1)}$ along the action map via gradient descent until reaching the initial point $\hat{p}_{0}$. For single branch detection, we may use this simplified process with an initial point located at one end of the tubular structure.

\subsection{Potentials}

The potential $\widetilde{P}_{k}$ is designed as a measurement which incorporates the full set of image values within the sphere surrounding the corresponding image point. Any sphere $s p$ in the image domain $\Omega_{I}$ is defined as a point $\hat{p}$ in $\Omega_{I_{r}}, s p=(p, r)$, where $p$ is the center point and $r$ is the radius. The entire sphere should lie inside the desired object and be as big as possible (so that it is tangential to the object boundary). Such spheres should exhibit lower values of $\widetilde{P}_{k}$ compared to smaller spheres which lie inside the desired object or to any sphere which lies outside (fully or partially) the desired object.

For any image point $p$ with gray value $I(p)$ in an image $I$, we define the mean intensity value $\mu(s p)$ and variance $\sigma^{2}(s p)$ for the sphere $s p=(p, r)$ as

$$
\mu(s p)=\frac{\int_{B(p, r)} I(\widetilde{p}) d \widetilde{p}}{\int_{B(p, r)} d \widetilde{p}}, \quad \sigma^{2}(s p)=\frac{\int_{B(p, r)}(I(\widetilde{p})-\mu(s p))^{2} d \widetilde{p}}{\int_{B(p, r)} d \widetilde{p}}
$$


where $B(p, r)$ represents the ball of radius $r$ centered at $p$. We propose an example potential

$$
\widetilde{P}_{k}(\hat{p})=\widetilde{P}_{k}(s p)=1 /\left(w+\lambda_{1}\left(\left|\mu\left(s p_{2}\right)-\mu(s p)\right|^{2}\right)+\lambda_{2}\left(\left|\sigma^{2}\left(s p_{2}\right)-\sigma^{2}(s p)\right|^{2}\right)\right),
$$

where $\mu\left(s p_{2}\right)$ and $\sigma^{2}\left(s p_{2}\right)$ represent the mean and the variance for the sphere $s p_{2}=$ $(p, 2 r), \omega$ is a real positive constant to control the smoothness of the obtained path, $\lambda_{1}$ and $\lambda_{2}$ are two real positive weights for the mean difference and variance difference between the detected sphere and a bigger sphere which has the same center point but twice the radius. These parameters should be selected based on the size and interior information of detected vessels, image noise levels, etc.

The potential shown in Eq. 8 considers the mean and variance differences between the detected sphere and the bigger sphere which shares the same center point with the detected sphere but has a bigger radius. It is irrelevant to $k$. If a sphere's radius is larger or smaller than the width of the tubular structure, the mean and variance differences between this sphere and the bigger sphere will decrease, and the related potential $\widetilde{P}_{k}$ will increase. This potential gives smallest values on spheres when they are exactly with center on centerline and the radii are as half as the width of the detected vessel. Also, this region based potential does not consider the difference between the current sphere and the starting sphere which therefore helps to avoid accumulation of detection errors along the fast gradient descent.

\section{Experimental Results and Analysis}

In this section, we demonstrate our approach on various $2 \mathrm{D}$ and $3 \mathrm{D}$ real images. For each test, users need to specify the center location and radius of the starting point, the potential, and the largest allowed radius of the tubular object. Users also need to specify the parameters $d_{\text {max }}, \widetilde{d}$, and $d_{\text {step }}$ for the 4D freezing fast marching scheme. Furthermore, all the results shown in this paper were processed on the original image data (i.e. no pre-processing steps were applied beforehand).
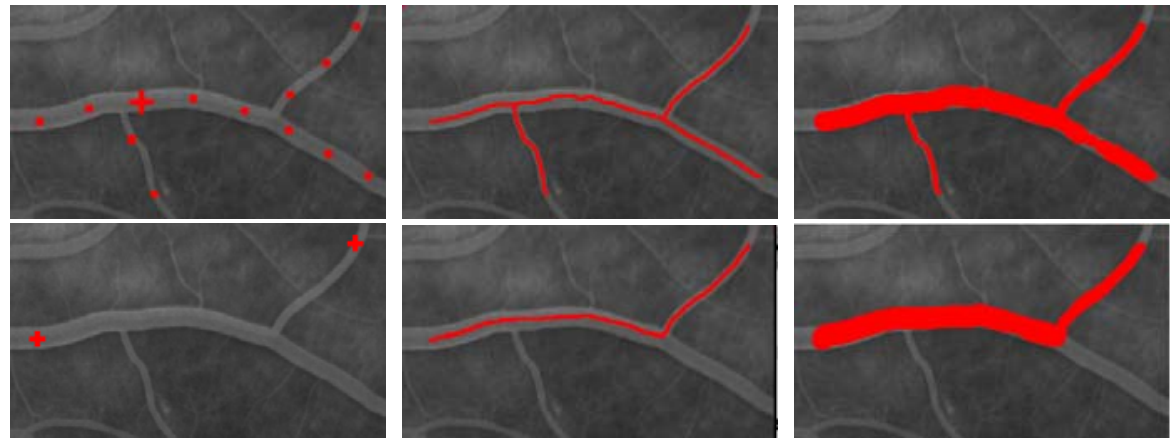

Fig. 2. Vessel segmentation for an angiogram $2 \mathrm{D}$ projection image based on the proposed method (upper row) versus the 4D minimal path method [14] (lower row). The initial point is shown with the red cross. Panels from left to right show the initial point and the detected iterative key points, the detected multi-branch centerlines, and the detected vessel surfaces. 

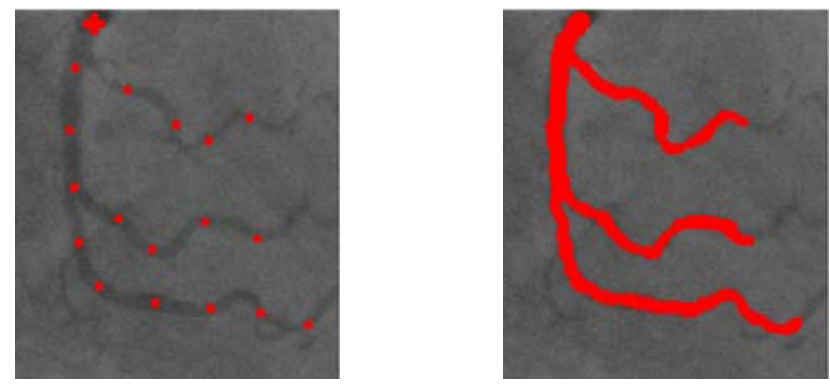

Fig. 3. Segmentation results via the proposed method on another 2D projection angiogram image. Panels from left to right show the initial point and the detected iterative key points and the detected vessel surfaces.

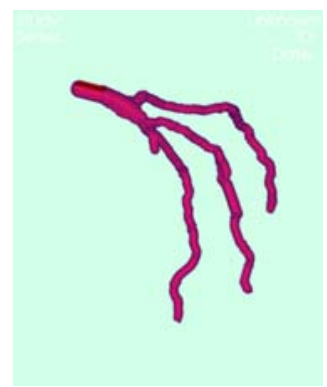

(a)

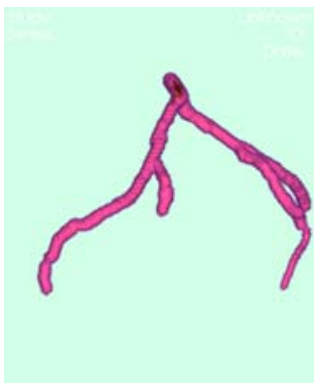

(b)

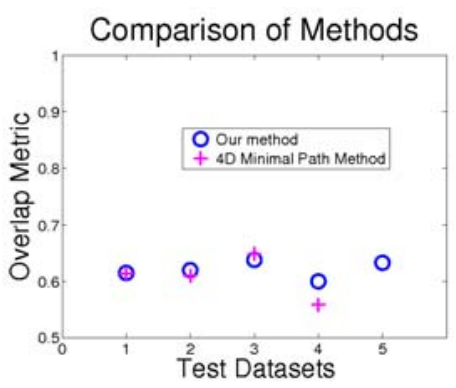

(c)

Fig. 4. 3D segmentation results shown for two out of five real 3D CTA data sets, plus comparisons for all five sets between the proposed method versus the 4D minimal path method [14] in terms of overlap with an expert's manual segmentations

Be begin in Fig. 2 with a 2D segmentation result for a noisy 2D projection angiogram image. We set $r_{\max }=15, r_{\text {initial }}=9, d_{\max }=0, \widetilde{d}=100$, and $d_{\text {step }}=50$ for the test. The initial point is located in the middle of the vessel structure. The potential is defined by Eq. 8 with $\omega=10, \lambda_{1}=10$, and $\lambda_{2}=10$. The result shows that by utilizing our 4D iterative key point scheme, multiple branches can be detected only with one initial point. For comparison, we also present the segmentation result on the same image based on the 4D minimal path method [14] which requires two input points instead of one and is incapable of branching. Fig. 3 shows an additional segmentation result with our algorithm on another 2D MRA projection image. In Fig. 4 , we test the iterative key point scheme on two 3D CTA datasets of the coronary artery. The potential is defined by Eq. 8 with $\omega=10, \lambda_{1}=10$, and $\lambda_{2}=10$. In these two tests, the initial points are located at the top of the left coronary artery.

Also, we show the comparison between the results obtained from our method (which required only one initial seed point per experiment) and the 4D minimal path method in [14] (which required 5 initial seed points per experiment in order to separately detect each branch). The comparison was done via overlap with manually aquired segmentations for 
the five 3D clinical CTA coronary datasets. No preprocessing was applied to the date for any of these experiments. Overlap Metric, $O M=2 \cdot \frac{N_{B} \cap N_{R}}{N_{B}+N_{R}}$, is applied to evaluate the detection accuracy, in which $N_{R}$ and $N_{B}$ are the number of reference ground truth voxels and the number of the detected voxels. For this comparison, we cropped a small region in which the vessel structures were detected by both our method and 4D minimal path method. The comparison illustrates that the proposed method can receive better results as the 4D minimal path technique with far less initial input demanded from the user.

\section{Conclusions}

In this paper, we proposed an innovative approach to extract multi-branch tubular structures using minimal user input. First, a novel 4D iterative key point searching method is proposed and utilized to detect multi-branch tubular structures with only one initial point. In contrast to standard minimal path techniques which require two initial points and can only detect one single branch between them, this new approach may propagate a tubular surface from only an initial seed point and detect 3D multi-branch surfaces and their centerlines simultaneously. Second, the freezing fast marching method is extended to 4D to reduce the computational cost of the iterative key point search. Finally, the designed potential, which is recalculated each time that a new key point is obtained, can avoid the path difference accumulation along a long tubular structure. It is a novel design and can be implemented easily in the iterative key point searching procedure.

\section{References}

1. Frangi, A., Niessen, W., Hoogeveen, R., van Walsum, T., Viergever, M.: Model-based Quantitation of 3D Magnetic Resonance Angiographic Images. IEEE Transactions on Medical Imaging 18(10), 946-956 (1999)

2. Duncan, J., Ayache, N.: Medical Image Analysis: Progress over Two Decades and the Challenges Ahead. IEEE Transactions on Pattern Analysis and Machine Intelligence 22(1), 85105 (2000)

3. Kirbas, C., Quek, F.: A Review of Vessel Extraction Techniques and Algorithms. ACM Computing Surveys (CSUR) 36(2), 81-121 (2004)

4. Sato, Y., Nakajima, S., Shiraga, N., Atsumi, H., Yoshida, S., Koller, T., Gerig, G., Kikinis, R.: Three-dimensional Multi-scale Line Filter for Segmentation and Visualization of Curvilinear Structures in Medical Images. Medical Image Analysis 2(2), 143-168 (1998)

5. Lorigo, L., Faugeras, O., Grimson, W., Keriven, R., Kikinis, R., Nabavi, A., Westin, C.: CURVES: Curve Evolution for Vessel Segmentation. Medical Image Analysis 5(3), 195-206 (2001)

6. Aylward, S., Bullitt, E.: Initialization, Noise, Singularities, and Scale in Height Ridge Traversal for Tubular Object Centerline Extraction. IEEE Transactions on Medical Imaging 21(2), 61-75 (2002)

7. Wink, O., Niessen, W., Viergever, M.: Multiscale Vessel Tracking. IEEE Transactions on Medical Imaging 23(1), 130-133 (2004)

8. Chung, A., Noble, A., Summers, P.: Vascular Segmentation of Phase Contrast Magnetic Resonance Angiograms Based on Statistical Mixture Modeling and Local Phase Coherence. IEEE Transactions on Medical Imaging 23(12), 1490-1507 (2004) 
9. Bouix, S., Siddiqi, K., Tannenbaum, A.: Flux Driven Automatic Centerline Extraction. Medical Image Analysis 9(3), 209-221 (2005)

10. Yan, P., Kassim, A.: Segmentation of Volumetric MRA Images by Using Capillary Active Contour. Medical Image Analysis 10(3), 317-329 (2006)

11. Deschamps, T., Cohen, L.: Fast Extraction of Minimal Paths in 3D Images and Applications to Virtual Endoscopy. Medical Image Analysis 5(4), 281-299 (2001)

12. Cohen, L., Kimmel, R.: Global Minimum for Active Contour Models: A Minimal Path Approach. In: IEEE International Conference on CVPR (CVPR 1996), pp. 666-673 (1996)

13. Sethian, J.: Fast Marching Methods. SIAM Review 41(2), 199-235 (1999)

14. Li, H., Yezzi, A.: Vessels as 4D Curves: Global Minimal 4D Paths to Extract 3D Tubular Surfaces and Centerlines. IEEE Transactions on Medical Imaging 26(9), 1213-1223 (2007)

15. Cohen, L., Deschamps, T.: Segmentation of 3D Tubular Objects with Adaptive Front Propagation and Minimal Tree Extraction for 3D Medical Imaging. Computer Methods in Biomechanics and Biomedical Engineering 10(4), 289-305 (2007) 